\title{
A Monte Carlo Model of the Neutron Detector Based on Lithium-Glass Scintillator
}

\author{
E. S. Kuzmin ${ }^{a, *}$, G. D. Bokuchava ${ }^{b}$, I. Yu. Zimin ${ }^{a}$, A. A. Kruglov ${ }^{b}$, \\ N. A. Kuchinskiy ${ }^{a}$, and V. L. Malyshev ${ }^{a}$ \\ ${ }^{a}$ Dzhelepov Laboratory of Nuclear Problems, Joint Institute for Nuclear Research (JINR), \\ Dubna, Moscow oblast, 141980 Russia \\ ${ }^{b}$ Frank Laboratory of Neutron Physics, Joint Institute for Nuclear Research (JINR), \\ Dubna, Moscow oblast, 141980 Russia \\ *e-mail:e_kuzmin@jinr.ru,e_kuzmin@mail.ru
}

Received August 4, 2020; revised August 20, 2020; accepted August 24, 2020

\begin{abstract}
A Monte Carlo model of the thermal-neutron scintillation detector based on NE 912 lithium glass has been created and verified. The simulation was validated by comparing its result to experimental data from a prototype detector exposed to thermal-neutron and $\gamma$-ray beams. The light yield in the scintillator for a captured thermal neutron, the quenching factor, and the decay times of the scintillator were determined. The accuracy in reproducing the pulse shapes obtained in the experiment is sufficient to allow analysis of experimental data and estimation of the efficiency of $n / \gamma$ discrimination techniques. Based on the simulation, it is possible to develop detector models with a low $\gamma$-ray sensitivity using heterogeneous composite scintillators with various geometries.
\end{abstract}

DOI: $10.1134 / \mathrm{S} 0020441221010279$

\section{INTRODUCTION}

The neutron detection technique is one of the key issues in neutron research. The widespread ${ }^{3} \mathrm{He}$-based detectors are becoming less available due to constraints in gas supply. Alternative types of detectors are required that can provide reasonable detection efficiency for neutrons in a wide energy range, a limited sensitivity to $\gamma$ rays or ability to discriminate them, and a short dead time. The known scintillation materials cannot meet all these requirements. The widely used ${ }^{6} \mathrm{LiF} / \mathrm{ZnS}(\mathrm{Ag})$ scintillation screen transmits its own radiation poorly and has a long dead time, i.e., approximately $2.5 \mu \mathrm{s}$ [1]. New neutron scintillators developed in recent years are expensive and can be manufactured only in small volumes [2]. Therefore, interest in wellknown lithium glasses has been awakened.

Lithium glasses have high transparency, high efficiency, short decay time, and, simultaneously, high sensitivity to $\gamma$ rays. Works on reducing the $\gamma$-ray sensitivity of glass scintillators are underway in two main directions. The first direction is to create heterogeneous scintillators based on lithium glasses with various geometries and optical characteristics, in particular, for problems of control over nuclear-material handling [3-5]. The second direction is to search for methods of digital signal processing to discriminate between pulses from neutrons and $\gamma$ rays $[6,7]$.
Development of a modern detector with a reduced $\gamma$-ray sensitivity calls for the algorithm for digital simulation of the detector structure and the methods for signal processing. In this paper, we describe a Monte Carlo model of the detector based on a homogeneous ${ }^{6} \mathrm{Li}$-enriched NE 912 glass scintillator and an XP2262 photomultiplier tube (PMT). The purpose of this simulation is to determine the scintillator parameters more precisely and to create a tool for developing detectors with a reduced sensitivity to $\gamma$ rays.

In this paper, we consider a thermal-neutron detector. The geometric shape, a reflector, a light guide, and optical contacts were taken into account in the simulation. The scintillator used in this study has not been produced for many years; however, numerous highquality devices have been in operation until now; e.g., see $[8,9]$. The characteristics of these detectors can be significantly improved using modern methods for reducing the $\gamma$-ray sensitivity. The model was verified by exposing the detector to a thermal-neutron beam from the IBR-2 reactor and to $\gamma$ rays from a ${ }^{60} \mathrm{Co}$ source.

The scintillation pulse was simulated using the Geant4 program in accordance with the detector design. The optical signal was convolved with a singleelectron pulse of the detector to calculate the output signal of the PMT. The simulated signals were then compared to experimental data. 


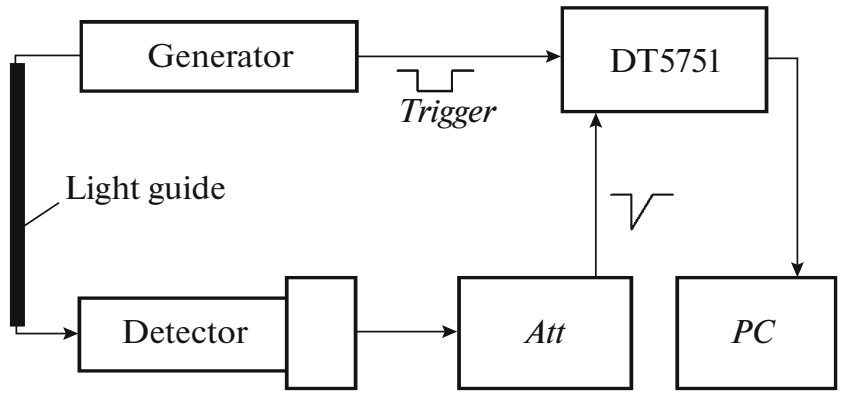

Fig. 1. The diagram of the PMT calibration. The light pulse from the generator is fed through the light guide to the entrance window of the PMT. The signal from the PMT anode and the trigger signal from the generator of light pulses are fed to the inputs of a 10-bit CAEN DT5751 waveform digitizer through $(A t t)$ attenuator; $(P C)$ personal computer.

\section{THE DETECTOR}

The detector based on NE 912 lithium glass scintillator (Nuclear Enterprises) and an XP2262B PMT (Photonis) with an S563/L voltage divider (Philips) was used in the study. The linearity of PMT response in the current range used in the measurements is $2 \%$ or better according to the manufacturer's data. A scintillator with a diameter of $40 \mathrm{~mm}$ and thickness of $2 \mathrm{~mm}$ was coupled to a PMT via a cylindrical Plexiglas light guide $40 \mathrm{~mm}$ in diameter and $25 \mathrm{~mm}$ in length. Optical contacts between the light guide and the scintillator and between the light guide and the entrance window of the PMT were provided by BC-630 optical grease (Bicron). The light guide coupled to the scintillator was placed in a fluoroplastic cylinder. The entrance window of the detector was located on the scintillator surface; it consisted of sequential diffuse and mirror reflectors with a total thickness of $0.08 \mathrm{~g} / \mathrm{cm}^{2}$.

The absolute calibration of the PMT with the spectrometric channel was carried out according to the method described in $[10,11]$. The method consists in analyzing the spectra recorded when the photocathode was illuminated with low-intensity light flashes (Fig. 1). The flash energy was controlled by the amplitude of the pulse applied to the LED; the duration of the rectangular pulse was $30 \mathrm{~ns}$ at a frequency of $1 \mathrm{kHz}$. The amplitude of the output PMT signal was regulated by a coaxial attenuator. The signals were recorded with a CAEN DT5751 10-bit analog-to-digital converter (ADC) using an external trigger from a flash generator. The gate width for data acquisition was $500 \mathrm{~ns}$, and gate began $250 \mathrm{~ns}$ before the trigger. The interval of signal integration was $100 \mathrm{~ns}$; it started $50 \mathrm{~ns}$ earlier than the trigger signal arrived.

Deconvolution of such spectra is performed using the response function constructed in [12]:

$$
\begin{aligned}
f(x)= & \sum_{n=0}^{\infty} \frac{\mu^{n} e^{-\mu}}{n !}\left\{\frac{(1-w)}{\sigma_{n} \sqrt{2 \pi}} \exp \left[\frac{\left(x-n Q_{1}-Q_{0}\right)^{2}}{-2 \sigma_{n}^{2}}\right]\right. \\
& \left.+w \theta\left(x-Q_{0}\right) \alpha \exp \left[-\alpha\left(x-Q_{0}\right)\right]\right\} .
\end{aligned}
$$

Expression (1) presents the following components of the experimental spectrum:

- the pedestal that characterizes the background processes of the first type. It describes the noise in the measurement section when no photoelectron is produced at the photocathode (e.g., fluctuations in the anode current). The pedestal is described by a normal distribution with mathematical expectation $Q_{0}$ and a standard deviation $\sigma_{0}$;

- the contributions from spectra with $n$ photoelectrons per pulse. Each spectrum is described by a normal distribution with mathematical expectations $Q_{n}$ and standard deviation $\sigma_{n}$;

- background processes of the second type, which are discrete noises. The contribution of such processes is described by a decreasing exponent with constant $\alpha$.

The symbol $\mu$ denotes the mean number of photoelectrons collected on the first dynode; the probability of the measured signal being accompanied by the second-type background processes is denoted as $w$; and $\theta(x)$ is the Heaviside function. In this case,

$$
Q_{n}=Q_{0}+n Q_{1} \quad \text { and } \quad \sigma_{n}=\sqrt{n} \sigma_{1}
$$

The fitting results are shown in Fig. 2.

The PMT was calibrated using a single-photoelectron spectrum; the first moment of the spectrum was determined with an accuracy of $0.4 \%$.

\section{EXPERIMENTAL MEASUREMENTS}

The neutron measurements were performed on the beam of the FSS diffractometer (channel 13 of the IBR-2 reactor) [13]. The experimental setup is shown in Fig. 3. When the detector was exposed to a thermalneutron beam, it was enclosed in a protective chamber made of lead and borated polyethylene. The detector was installed at an angle of $42^{\circ}$ with the beam axis. The primary neutron beam was scattered from a $20-\mathrm{mm}$ diameter target made of reactor graphite, which was free from admixtures of boron isotopes that generated $\gamma$ rays during thermal-neutron capture.

A CAEN DT5751 ADC was directly connected to the anode output of the PMT via a 1.5-m-long cable. An internal trigger with a threshold of $45 \mathrm{mV}$ was used in the measurements. The gate for the recording was $2000 \mathrm{~ns}$ long and started $280 \mathrm{~ns}$ before the trigger. The bias voltage was supplied from the NDT 1470 powersupply module (CAEN). The ADC and the bias-voltage source, as well as the data acquisition process were controlled by an intermediate computer located in 


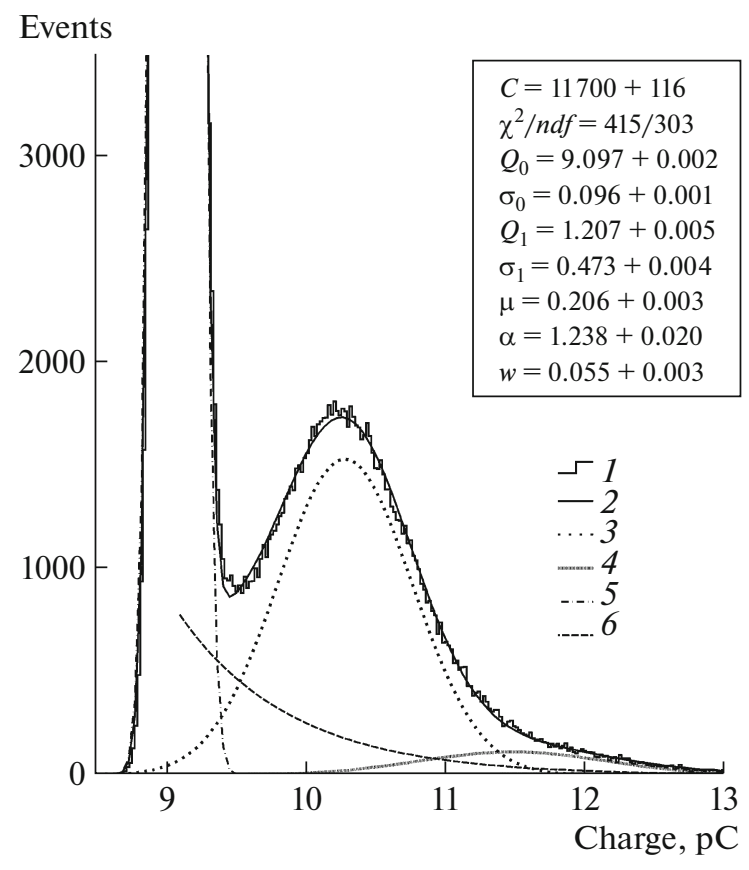

Fig. 2. The spectrum of pulses measured during PMT calibration: (1) measurement data, (2) result of fitting according to Eq. (1), (3) distribution of charges from a single electron, (4) distribution of charges from two and three photoelectrons, (5) pedestal, and (6) background processes of the second type.

close proximity to the detector. The general control of the experiment was exerted from a remote computer via the Internet. The mean count rate for neutrons was at a level of $600 \mathrm{~s}^{-1}$.

The measurements with the ${ }^{60} \mathrm{Co}$ radioactive source were carried out using a similar technique. The source with an activity of $46.3 \mathrm{kBq}$ in a mandrel was located at the entrance window of the detector at a distance of $16.0 \mathrm{~mm}$ from the front surface of the scintillator. The configuration of the electronic system and the detection parameters were the same as for the neutron detection. The mean count rate for $\gamma$ rays was at a level of $500 \mathrm{~s}^{-1}$.

When the signal was integrated, the processing gate began $30 \mathrm{~ns}$ before the trigger; its duration was $450 \mathrm{~ns}$. The front-end signal processing was performed in three stages. The baseline selection was carried out at the first stage. Since the baseline should not change its value in the absence of noise, the following limitation was set: if the baseline value for the initial 250 ns differed by more than $0.5 \%$, such a signal was rejected. Selection according to the position of the pulse peak was the second stage. When the internal trigger was used, the position of the signal relative to the processing gate had to be constant. When the signal was displaced for a greater distance from the beginning of the gate, the event was not used due to incomplete signal

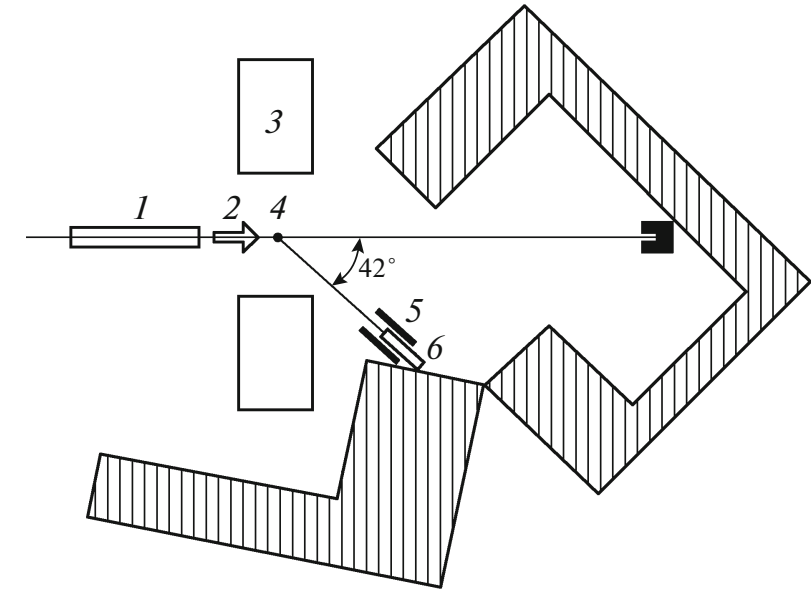

Fig. 3. The diagram of the experiment with the thermal neutron beam: (1) neutron guide, (2) a beam of thermal neutrons, (3) auxiliary equipment, (4) reactor graphite diffuser, (5) detector protection, (6) detector.

integration. At the third stage, events were sorted by duration. The noise signals from the PMT were significantly shorter than the signals from the scintillator; therefore, events with a full width at half-maximum FWHM $<20$ ns were rejected. The spectra obtained as a result of processing are shown in Fig. 4.

\section{THE CHARACTERISTICS OF LIGHT EMISSION OF THE SCINTILLATOR}

The construction of a digital detector model and the study of the possibilities of discriminating between neutron and $\gamma$-ray signals require information on the characteristics of light emission of the scintillator. Such information can only partially be found in the literature [14]. The mean shape of the scintillator pulse from neutrons and $\gamma$ rays was constructed by adding 400 pulses with similar amplitudes for each type of excitation. The superposition of pulses on the time scale was carried out at a point corresponding to the middle of the leading edge. The resulting pulse shapes were used to determine the luminescence parameters of the scintillator in a time interval of 50-450 ns.

The shape of a signal from a scintillation detector is determined by the convolution of the emission function of the scintillator with the response function of the PMT and the data-acquisition system [15].

For the case of one decay-time constant, the pulse shape can be represented by the difference of two exponential terms, one of which corresponds to the equivalent time constant of the anode circuit $R C$, the connecting cable, and the input circuits of the electronics, and the other corresponds to the decay time of the scintillator.

In our case, the scintillator has two fast luminescence components and one slow component that 


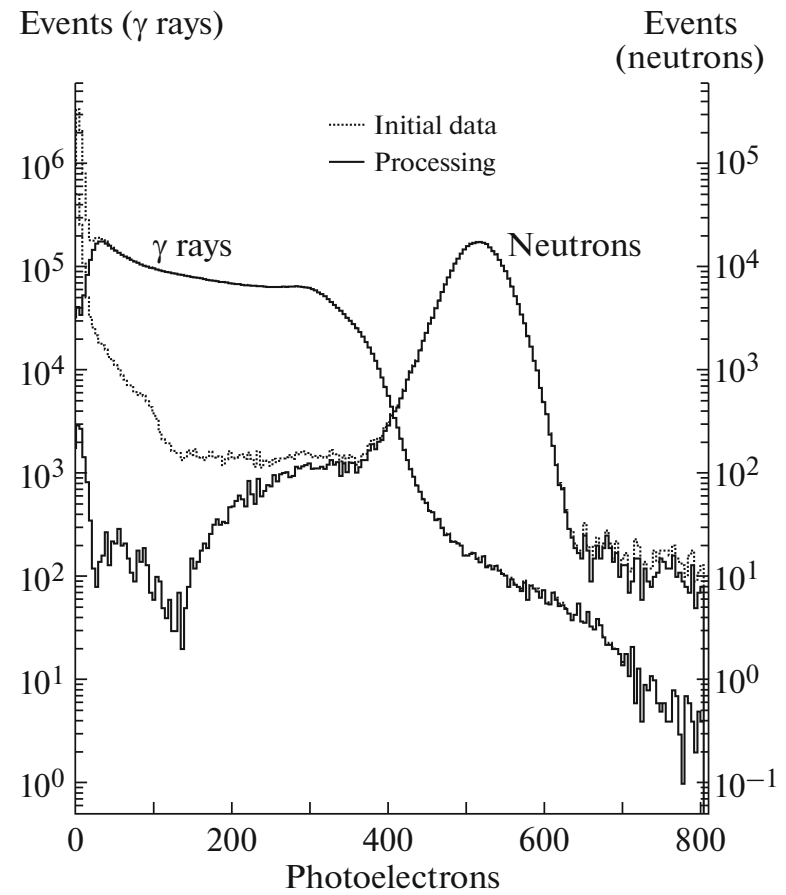

Fig. 4. The spectra of signals from ${ }^{60} \mathrm{Co}$ and thermal neutrons before and after the processing. The spectra are superimposed on an axis calibrated in terms of photoelectrons.

describes the afterglow; therefore, the pulse shape can be described by the sum of four terms:

$$
\begin{gathered}
L=A\left\{\exp \left[-\theta\left(t-t_{0}\right)\right]-\exp \left[-\lambda_{1}\left(t-t_{0}\right)\right]\right\} \\
+B\left\{\exp \left[-\theta\left(t-t_{0}\right)\right]-\exp \left[-\lambda_{2}\left(t-t_{0}\right)\right]\right\} \\
+C\left\{\left[-\theta\left(t-t_{0}\right)\right]-\exp \left[-\lambda_{3}\left(t-t_{0}\right)\right]\right\},
\end{gathered}
$$

where $\lambda=1 / \tau$ is the decay constant of the scintillator; $\lambda_{1}$ and $\lambda_{2}$ are the fast components; $\lambda_{3}$ is the afterglow; $\theta$ is the time constant of the anode; $t_{0}$ is the pulse start time; $A, B$, and $C$ are the amplitudes of the lumines-

Table 1. The light emission characteristics of the NE 912 scintillator upon excitation by thermal neutrons and $\gamma$ rays: our experiment and the data from [14]

\begin{tabular}{l|c|c|c}
\hline \multicolumn{3}{c}{ Neutrons } \\
\hline Component number & 1 & 2 & 3 \\
Amplitude (experiment) & 0.39 & 0.53 & 0.08 \\
Decay time, ns (experiment) & 15 & 56 & 303 \\
Decay time, ns [14] & 18 & 55 & - \\
\hline \multicolumn{3}{c}{$\gamma$ rays } \\
\hline Component number & 1 & 2 & 3 \\
Amplitude (experiment) & 0.54 & 0.43 & 0.03 \\
Decay time, ns (experiment) & 40 & 84 & 867 \\
\hline
\end{tabular}

Amplitude, $\mathrm{mV}$
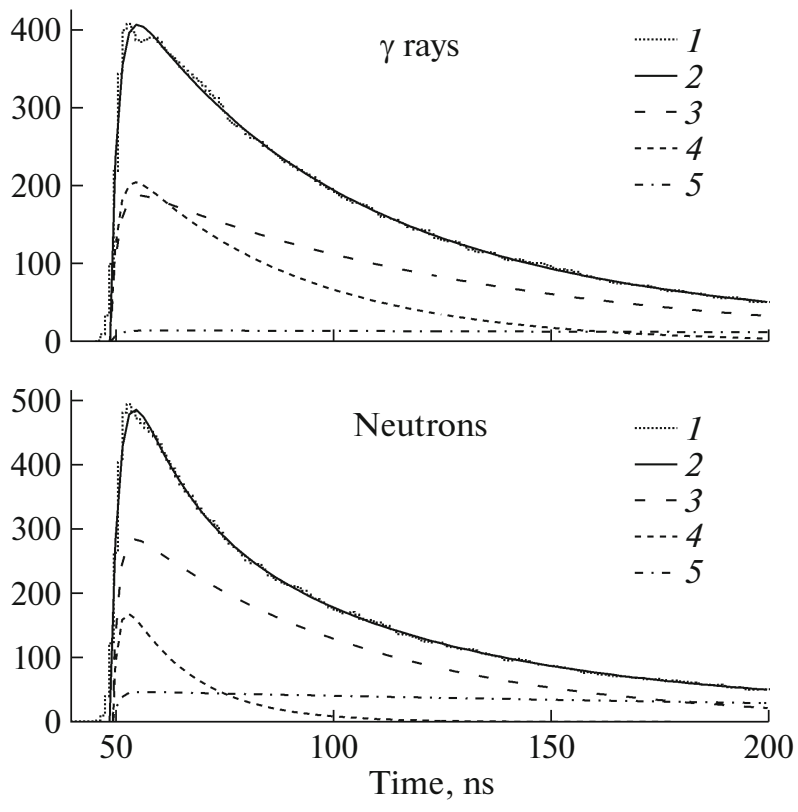

Fig. 5. The averaged pulses recorded in the experiment when the scintillator was excited by $\gamma$ rays and neutrons: (1) averaged shape of the recorded pulses, (2) result of fitting according to Eq. (2), (3) slow scintillation component, (4) fast scintillation component, and (5) afterglow.

cence components. The detector pulses averaged for different types of excitation were fitted according to Eq. (2). The values of the decay times and amplitudes obtained by fitting are presented in Table 1 in comparison with literature data [14] and are illustrated in Fig. 5.

\section{THE SIMULATION OF THE DETECTOR}

The Monte Carlo simulation of the energy spectra and signals from the detector under exposure to thermal neutrons and $\gamma$ rays was performed using the Geant4 version 10.06.p01 software package [16]. Several standard software packages of physical processes developed at CERN for simulating detectors were also used. Geant 4 allows one to simulate the entire process of particle passing through the detector, the production of light photons, and their passage through detector components. Geant 4 includes a number of packages for simulating electromagnetic interactions of particles with matter, which are specialized for different particle types, energy ranges, and used physical models. In our study, we consider only $\gamma$ rays and thermal neutrons and use the standard G4EmStandardPhysics_option4 package of electromagnetic processes and the G4HadronPhysicsQGSP_BERT_HP package of inelastic processes (in particular, for neutrons with energies as high as $20 \mathrm{MeV}$ ).

Based on the initial spectrum, the algorithm determined the total energy deposited in the scintillator by 


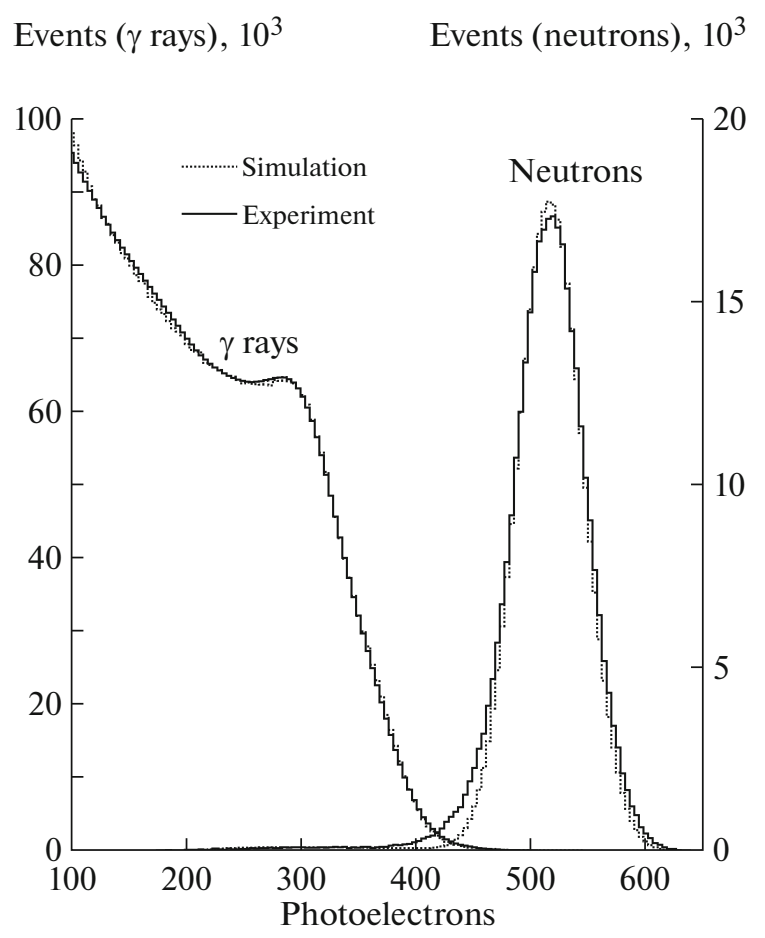

Fig. 6. The comparison of the simulated and measured spectra of events due to $\gamma$ rays from ${ }^{60} \mathrm{Co}$ and thermal neutrons, which are superimposed on an axis calibrated in terms of photoelectrons.

each particle. Light pulses from neutrons and $\gamma$ rays were generated using the amplitudes and decay times of the scintillator from Table 1. The total number of photoelectrons in the pulse was used to construct the charge spectrum of events.

The geometric characteristics of the detector, absorption lengths, and optical properties of the interfaces were used to simulate the transmission of optical photons, including scattering, absorption, reflection, and refraction at the boundaries of optical media. The UNIFIED model was used to simulate the behavior of photons at the interfaces. The model takes all aspects of treatment of surfaces and reflective coatings into account. The comparison of the experimental spectrum with the simulation result for $\gamma$ rays from the ${ }^{60} \mathrm{Co}$ source and thermal neutrons is shown in Fig. 6.

The general agreement between the experimental and simulated data for $\gamma$ rays is quite good, except for the region of low energy transfers. The difference is explained by the fact that the description of the setup geometry did not include auxiliary components of the source unit (the housing, plugs, fasteners, etc.), which distort the particle flux. Precise simulation of these components requires significant resources. Good agreement is observed for neutrons in the central region of the spectrum. The difference between the

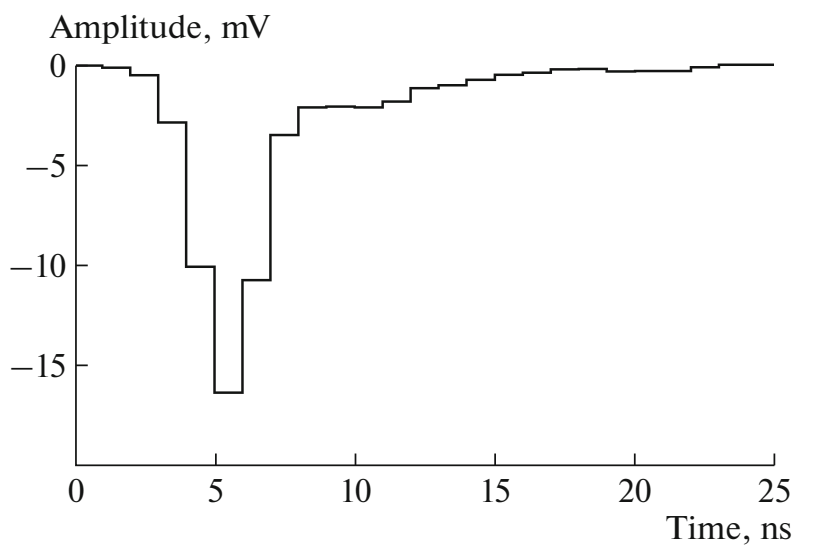

Fig. 7. The pulse shape at the detector output when a single photoelectron hits the first dynode.

experimental and simulated data in the region of low transferred energies results, in our opinion, from the inhomogeneity of the conversion efficiency over the scintillator volume.

The light yield in the NE 912 scintillator during detection of electrons was measured in [17]. Its value is $L_{e}=3257$ photons/MeV. The central part of spectra that were measured in the thermal neutron beam and obtained by simulation is described by a normal distribution. This fact has made it possible to precisely fit the model spectrum to the experimental one by varying the light yield in the scintillator under exposure to neutrons. The light yield determined in this manner for neutrons is $L_{n}=1048$ photons/MeV. The quenching factor obtained from these values for neutron detection is $\mathrm{QF}=0.32$.

The distribution of the number of photons at the photocathode was specified by recording the arrival time of each light photon at the photocathode after scintillation. This distribution was converted into a PMT signal in view of the single-photoelectron response function. The shape of the single-photoelectron pulse was obtained using a dataset recorded during PMT calibration. For this purpose, 2000 pulses with amplitudes located in a narrow range at the top of the single-electron peak were added together. The shape of such a pulse is shown in Fig. 7.

The output detector signal was formed in the simulation by performing the convolution of the light pulse arriving at the photocathode and the single-photoelectron signal [18]. The signals obtained by simulation were compared to the signals recorded in the experiment. The average simulated signals obtained by adding 400 unit pulses were used in the comparison.

The similarity or difference between signals is completely determined by their cross-correlation coefficient. The Pearson correlation coefficients were calculated for 1500 signals recorded in the experiment for both thermal neutrons and $\gamma$ rays in order to estimate 

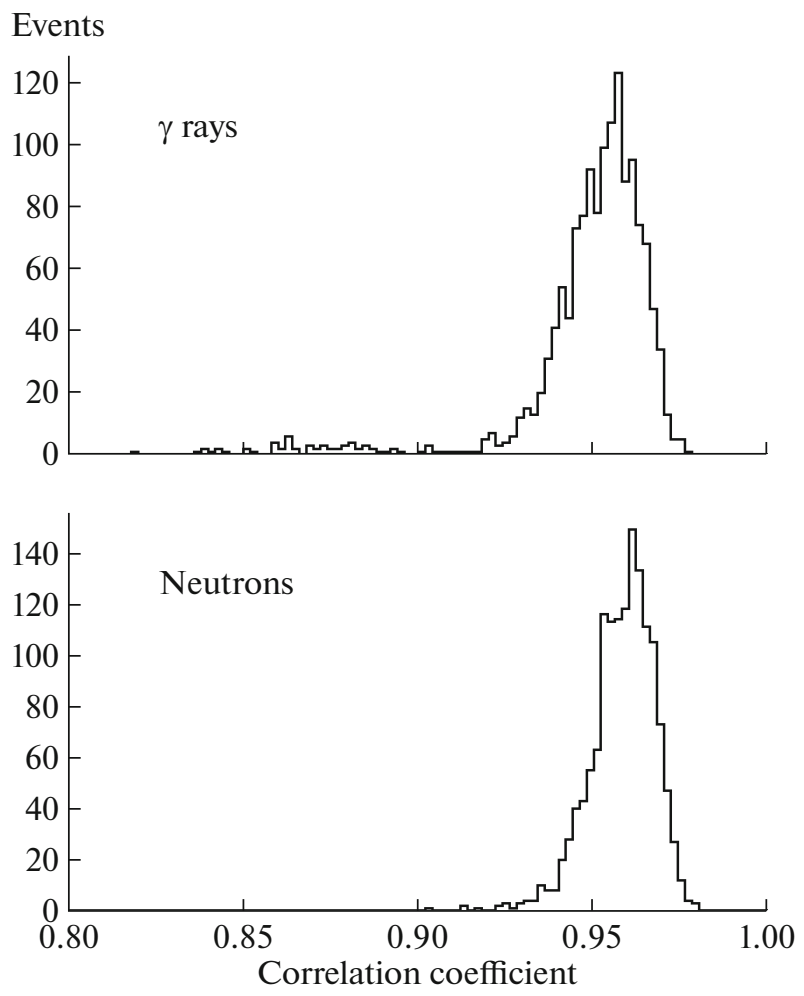

Fig. 8. The distribution of Pearson correlation coefficients for $\gamma$ rays and neutrons.

the similarity between the average simulated pulse and the set of experimental signals. The distributions of the correlation coefficients are shown in Fig. 8.

The result demonstrates a high degree of similarity between the model and experimental pulses. The average value of the correlation coefficient is 0.96 for neutrons and 0.95 for $\gamma$ rays. A number of experimental pulses show a low degree of similarity with the model pulse due to statistical fluctuations.

\section{CONCLUSIONS}

The Monte Carlo model of a scintillation detector of thermal neutrons based on NE 912 lithium glass was created and verified. The characteristics of the scintillator were determined: the light yield for neutrons, the quenching factor, and the decay times of scintillation. The spectra obtained for neutrons and $\gamma$ rays using the Monte Carlo method generally agree well with the experimental data. All the characteristic features of the experimental distributions were reproduced in the simulation, and the relative intensities of the different spectral regions were in good agreement. The accuracy in reproducing of the shape of pulses recorded in the experiment makes it possible to investigate the $\gamma$-ray sensitivity of the detector using various methods of particle selection. Based on these results, it is possible to construct a detector model with a heterogeneous scintillator and develop digital methods for particle discrimination.

\section{REFERENCES}

1. Kuzmin, E.S., Balagurov, A.M., Bokuchava, G.D., Zhuk, V.V., and Kudryashev, V.A., J. Neutron Res., 2002, vol. 10, p. 31. https://doi.org/10.1080/10238160290027748

2. Combes, C.M., Dorenbos, P., Van Eijk, C.W.E., Krämer, K.W., and Güdel, H.U., J. Lumin., 1999, vol. 82, no. 4, p. 299.

3. Ianakiev, K.D., Hehlen, M.P., Swinhoe, M.T., Favalli, A., Iliev, M.L., Lin, T.C., Bennett, B.L., and Barker, M.T., Nucl. Instrum. Methods Phys. Res., Sect. A, 2015, vol. 784, p. 189. https://doi.org/10.1016/j.nima.2014.10.073

4. Mayer, M., Nattress, J., Trivelpiece, C., and Jovanovic, I., Nucl. Instrum. Methods Phys. Res., Sect. A, 2015, vol. 784, p. 168. https://doi.org/10.1016/j.nima.2014.09

5. Rich, G.C., Kazkaz, K., Martinez, H.P., and Gushue, T., Nucl. Instrum. Methods Phys. Res., Sect. A, 2015, vol. 794, p. 15. https://doi.org/10.1016/J.NIMA.2015.05.004

6. Zaitseva, N., Glenn, A., Martinez, H.P., Carman, L., Pawełczak, I., Faust, M., and Payne, S., Nucl. Instrum. Methods Phys. Res., Sect. A, 2013, vol. 729, p. 747. https://doi.org/10.1016/j.nima.2013.08.048

7. Wang, C.L. and Riedel, R., Rev. Sci. Instrum., 2016, vol. 87 , p. 013301 . https://doi.org/10.1063/1.4939821

8. Bokuchava, G., Crystals, 2018, vol. 8, p. 318. https://doi.org/10.3390/cryst8080318

9. Balagurov, A., Balagurov, D., Bobrikov, I., Bogdzel, A., Drozdov, V., Kirilov, A., Kruglov, V., Kulikov, S., Murashkevich, S., Prikhodko, V., Shvetsov, V., Simkin, V., Sirotin, A., Zernin, N., and Zhuravlev, V., Nucl. Instrum. Methods Phys. Res., Sect. B, 2018, vol. 436, p. 263.

10. Bellamy, E.H., Bellettini, G., Budagov, J., Cervelli, F., Chirikov-Zorin, I.E., Incagli, M., Lucchesi, D., Pagliarone, C.E., Tokár, S., and Zetti, F., Nucl. Instrum. Methods Phys. Res., Sect. A, 1994, vol. 339, p. 468. https://doi.org/10.1016/0168-9002(94)90183-X

11. Bellamy, E.H., Belletini, G., Budagov, J., Cervelli, F., Chiricov-Zorin, I.E., Kovtun, V., Incagli, M., Lucchesi, D., Pagliarone, C.E., Pukhov, J., Seminozhenko, V.P., Senchishin, V.S., Tokár, S., Verezub, N.A., Zljubovsky, I.I., and Zetti, F., Nucl. Instrum. Methods Phys. Res., Sect. A, 1994, vol. 334, p. 484. https://doi.org/10.1016/0168-9002(94)90228-3

12. Chirikov-Zorin, I.E., Cand. Sci. (Phys.-Math.) Dissertation, Dubna: Joint Institute for Nuclear Research, 2014. https://search.rsl.ru/ru/record/01007866157.

13. Schroder, J., Kudryashev, V.A., Keuter, J.M., Priesmeyer, H.G., Larsen, J., and Tiitta, A., J. Neutron Res., 1994, vol. 2, no. 4, p. 129.

https://doi.org/10.1080/10238169408200025 
14. Fairley, E.J. and Spowart, A.R., Nucl. Instrum. Methods, 1978, vol. 150, p. 159.

15. Marrone, S., Cano-Ott, D., Colonna, N., Domingo, C., Gramegna, F., Gonzalez, E.M., Gunsing, F., Heil, M., Kappeler, F., Mastinu, P.F., Milazzo, P.M., Papaevangelou, T., Pavlopoulos, P., Plag, R., Reifarth, R., Tagliente, G., Tain, J.L., and Wisshak, K., Nucl. Instrum. Methods Phys. Res., Sect. A, 2002, vol. 490, p. 299. https://doi.org/10.1016/S0168-9002(02)01063-X
16. Geant4, A Simulation Toolkit. https://geant4.web.cern.ch/support/download.

17. Dalton, A.W., Nucl. Instrum. Methods Phys. Res., Sect. $A$, 1987, vol. 254 , p. 361.

18. Haoyang Xing, Xunzhen Yu, Jingjun Zhu, Li Wang, Jinglu Ma, Shukui Liu, Linwei Li, Liejian Chen, Changjian Tang, and Qian Yue, Nucl. Instrum. Methods Phys. Res., Sect. A, 2014, vol. 768, p. 1.

https://doi.org/10.1016/J.NIMA.2014.08.049

Translated by N. Goryacheva 